\title{
The Distribution of Quadratic Expressions in Elliptically Contoured Vectors
}

\author{
Serge B. Provost ${ }^{1} \&$ Ali Akbar Mohsenipour ${ }^{1}$ \\ ${ }^{1}$ Department of Statistical \& Actuarial Sciences, The University of Western University Ontario, London, Ontario, \\ Canada \\ Correspondence: Serge B. Provost, Department of Statistical \& Actuarial Sciences, The University of Western \\ University Ontario, London, Ontario, Canada. Tel: 1-519-661-3624. E-mail: provost@stats.uwo.ca
}

Received: July 30, 2012 Accepted: August 13, 2012 Online Published: September 18, 2012

doi:10.5539/ijsp.v1n2p103 URL: http://dx.doi.org/10.5539/ijsp.v1n2p103

This research was supported by the Natural Sciences and Engineering Research Council of Canada

\begin{abstract}
A general representation of quadratic expressions in possibly singular elliptically contoured random vectors, as well as a procedure for the numerical evaluation of their distributions, are proposed in this paper. First, such quadratic expressions are represented as the difference of two positive definite elliptically contoured quadratic forms plus an independently distributed linear combination of spherically distributed random variables. Their distributions are then determined from a representation of elliptically contoured vectors in terms of scale mixtures of Gaussian vectors. Quadratic forms and quadratic expressions in various types of elliptically contoured vectors are considered. An accurate moment-based approximation to their density function is also provided. Several numerical examples illustrate the results.
\end{abstract}

Keywords: quadratic expressions, elliptically contoured vectors, spherically distributed vectors, quadratic forms, cumulant generating function, moments, density approximation

\section{Introduction}

Several fields of application involve elliptically contoured distributions, including, for instance, anomalous change detection in hyperspectral imagery: Theiler et al. (2010); option pricing: Hamada and Valdez (2008); filtering and stochastic control: Chu (1973); random input signal: McGraw and Wagner (1968); financial analysis: Zellner (1976) and the references therein; the analysis of stock market data: Mandelbrot (1963) and Fama (1965); and Bayesian Kalman filtering: Girón and Rojano (1994). Additionally, studies on the robustness of statistical procedures when the probability model departs from the multivariate normal distribution to the broader class of elliptically contoured distributions were carried out by King (1980) and Osiewalski and Steel (1993). Several multivariate applications are also discussed in Devlin et al. (1976). Results related to regression analysis can be found, for example, in Fraser and Ng (1980). Heavy-tailed time series models were discussed in Resnick (1997). A new family of life distributions, generated from an elliptically contoured distribution, is discussed by Díaz-García and Leiva-Sánchez (2005). Recently, Ip et al. (2007) derived some results applicable to Bayesian inference for a general multivariate linear regression model with matrix variate elliptically distributed errors. In fact, the class of elliptically contoured distributions, which contains the multivariate normal distribution, enjoys several of its properties while allowing for more flexibility in modeling various random processes.

A $p$-dimensional vector $\mathbf{X}$ has an ellipticallycontoured or elliptical distribution with mean vector $\boldsymbol{\mu}$ and scale parameter matrix $\Sigma$ if its characteristic function $\phi(\mathbf{t})$ can be written as

$$
\phi(\mathbf{t})=e^{i \mathbf{t}^{\prime} \boldsymbol{\mu}} \xi\left(\mathbf{t}^{\prime} \Sigma \mathbf{t}\right)
$$

where $\boldsymbol{\mu}$ is a $p$-dimensional real vector, $\Sigma$ is a $p \times p$ nonnegative definite matrix and $\xi(\cdot)$ is a nonnegative function, see, for instance, Cambanis et al. (1981); this will be denoted $\mathbf{X} \sim C_{p}(\mu, \Sigma ; \xi)$.

Moreover, the densities associated with $p$-dimensional elliptically contoured vectors $\mathbf{X}$ are of the form $h((\mathbf{x}-$ $\left.\boldsymbol{\mu})^{\prime} \Sigma^{-1}(\mathbf{x}-\boldsymbol{\mu})\right)$ where $h(\cdot)$ is a density defined on $(0, \infty)$ whose $(p / 2-1)^{\text {th }}$ moment exists, see for example Fang et 
al. (1990), Section 2.2.3. In particular, when $\boldsymbol{\mu}$ is the null vector and $\Sigma$ is the identity matrix of order $p, \mathbf{X}$ is said to have a spherically symmetric or spherical distribution; this will be denoted $\mathbf{X} \sim \mathcal{S}_{p}(\xi)$.

In fact, whenever $\mathbf{Y} \sim \mathcal{C}_{p}(\boldsymbol{\mu}, \Sigma ; \xi)$ and $\Sigma$ is a positive definite matrix, $\Sigma^{-\frac{1}{2}}(\mathbf{Y}-\boldsymbol{\mu}) \sim \mathcal{S}_{p}(\xi)$, where $\Sigma^{-1 / 2}$ denotes the inverse of the symmetric square root of $\Sigma$. Furthermore, spherical distributions are invariant under orthogonal transformations, that is, for any orthogonal matrix $P, \mathbf{X} \sim \mathcal{S}_{p}(\xi)$ and $P \mathbf{X}$ are identically distributed. Other characterizations and properties are available from Kelker (1970), Chmielewski (1981), Fang et al. (1990) and Mathai et al. (1995), among others.

A decomposition of quadratic expressions in possibly singular elliptically contoured vectors is introduced in Section 2 and representations of functions of elliptically contoured vectors such as the moments of a quadratic form, are obtained in Section 3. A density approximation methodology that combines these results is described and illustrated by several numerical examples in Section 4.

The distributional results derived in this paper for quadratic forms in elliptically contoured random vectors not only extend, but also make use of, their Gaussian counterparts. Given that elliptically contoured distributions are utilized as models in a host of applications, and quadratic forms are ubiquitous in statistics, the result presented herein should prove useful in a variety of contexts and lead to the development of improved statistical inference techniques.

\section{A Decomposition of Quadratic Expressions in Elliptically Contoured Vectors}

Consider the quadratic expression $Q^{*}(\mathbf{X})=(\mathbf{X}-\boldsymbol{\alpha})^{\prime} A(\mathbf{X}-\boldsymbol{\alpha})+\mathbf{a}^{\prime}(\mathbf{X}-\boldsymbol{\alpha})+d$ where $\mathbf{X} \sim \mathcal{C}_{p}(\boldsymbol{\mu}, \Sigma ; \xi), \operatorname{rank}(\Sigma)=r \leq p$, $\alpha$ is a $p$-dimensional real vector and $A$ is a real symmetric matrix. Letting $\mathbf{X}=\boldsymbol{\mu}+B \mathbf{S}$, where $B_{p \times r}$ is such that $B B^{\prime}=\Sigma$ (cf. Example 4) and $\mathbf{S} \sim \mathcal{S}_{r}(\xi)$, one can write

$$
\begin{aligned}
Q^{*}(\mathbf{X}) \equiv Q^{*}(\mathbf{S}) & =(\boldsymbol{\mu}+B \mathbf{S}-\boldsymbol{\alpha})^{\prime} A(\boldsymbol{\mu}+B \mathbf{S}-\boldsymbol{\alpha})+\mathbf{a}^{\prime}(\boldsymbol{\mu}+B \mathbf{S}-\boldsymbol{\alpha})+d \\
& =[(\boldsymbol{\mu}-\boldsymbol{\alpha})+B \mathbf{S}]^{\prime} A[(\boldsymbol{\mu}-\boldsymbol{\alpha})+B \mathbf{S}]+\mathbf{a}^{\prime}[(\boldsymbol{\mu}-\boldsymbol{\alpha})+B \mathbf{S}]+d \\
& =\boldsymbol{\mu}_{1}^{\prime} A \boldsymbol{\mu}_{1}+2 \boldsymbol{\mu}_{1}^{\prime} A^{\prime} B \mathbf{S}+\mathbf{S}^{\prime} B^{\prime} A B \mathbf{S}+\mathbf{a}^{\prime} B \mathbf{S}+\mathbf{a}^{\prime} \boldsymbol{\mu}_{1}+d
\end{aligned}
$$

where $\mu_{1}=\boldsymbol{\mu}-\boldsymbol{\alpha}$. Let $P$ be an orthogonal matrix such that $P^{\prime} B^{\prime} A B P=\operatorname{Diag}\left(\lambda_{1}, \ldots, \lambda_{r}\right), \lambda_{1}, \ldots, \lambda_{r}$ denoting the eigenvalues of $B^{\prime} A B$, with $\lambda_{1}, \ldots, \lambda_{r_{1}}$ positive, $\lambda_{r_{1}+1}=\cdots=\lambda_{r_{1}+\theta}=0$ and $\lambda_{r_{1}+\theta+1}, \ldots, \lambda_{r}$ negative, $\mathbf{m}^{\prime}=\left(m_{1}, \ldots, m_{r}\right)=\mathbf{a}^{\prime} B P, \quad \mathbf{b}^{*^{\prime}}=\left(b_{1}^{*}, \ldots, b_{r}^{*}\right)=\boldsymbol{\mu}_{1}^{\prime} A B P, c_{1}=\boldsymbol{\mu}_{1}^{\prime} A \boldsymbol{\mu}_{1}+\mathbf{a}^{\prime} \boldsymbol{\mu}_{1}+d$. Then, letting $\mathbf{W}=\left(W_{1}, \ldots, W_{r_{1}}, \ldots, W_{r_{1}+\theta+1}, \ldots, W_{r}\right)^{\prime}=P^{\prime} \mathbf{S} \sim \mathcal{S}_{r}(\xi)$ and assuming that $B^{\prime} A B \neq O$, one has

$$
\begin{aligned}
Q^{*}(\mathbf{X}) \equiv Q^{*}(\mathbf{W})= & \mathbf{W}^{\prime} P^{\prime} B^{\prime} A B P \mathbf{W}+2 \boldsymbol{\mu}_{1}^{\prime} A B P \mathbf{W}+\mathbf{a}^{\prime} B P \mathbf{W}+\boldsymbol{\mu}_{1}^{\prime} A \boldsymbol{\mu}_{1}+\mathbf{a}^{\prime} \boldsymbol{\mu}_{1}+d \\
= & \mathbf{W}^{\prime} \operatorname{Diag}\left(\lambda_{1}, \ldots, \lambda_{r}\right) \mathbf{W}+\left(2 \mathbf{b}^{*^{\prime}}+\mathbf{m}^{\prime}\right) \mathbf{W}+c_{1} \\
= & \sum_{j=1}^{r_{1}} \lambda_{j} W_{j}^{2}+2 \sum_{j=1}^{r_{1}} n_{j} W_{j}-\sum_{j=r_{1}+\theta+1}^{r}\left|\lambda_{j}\right| W_{j}^{2}+2 \sum_{j=r_{1}+\theta+1}^{r} n_{j} W_{j} \\
& +2 \sum_{j=r_{1}+1}^{r_{1}+\theta} n_{j} W_{j}+c_{1} \\
= & \sum_{j=1}^{r_{1}} \lambda_{j}\left(W_{j}+\frac{n_{j}}{\lambda_{j}}\right)^{2}-\sum_{j=r_{1}+\theta+1}^{r}\left|\lambda_{j}\right|\left(W_{j}+\frac{n_{j}}{\lambda_{j}}\right)^{2}+2 \sum_{j=r_{1}+1}^{r_{1}+\theta} n_{j} W_{j} \\
& +\left(c_{1}-\sum_{j=1}^{r_{1}} \frac{n_{j}^{2}}{\lambda_{j}}-\sum_{j=r_{1}+\theta+1}^{r} \frac{n_{j}^{2}}{\lambda_{j}}\right) \\
\equiv & Q_{1}\left(\mathbf{W}^{+}\right)-Q_{2}\left(\mathbf{W}^{-}\right)+2 \sum_{j=r_{1}+1}^{r_{1}+\theta} n_{j} W_{j}+\kappa_{1} \\
\equiv & Q_{1}\left(\mathbf{W}^{+}\right)-Q_{2}\left(\mathbf{W}^{-}\right)+T_{1},
\end{aligned}
$$

where $Q_{1}\left(\mathbf{W}^{+}\right)=\mathbf{W}^{+^{\prime}} \operatorname{Diag}\left(\lambda_{1}, \ldots, \lambda_{r_{1}}\right) \mathbf{W}^{+}$and $Q_{2}\left(\mathbf{W}^{-}\right)=\mathbf{W}^{-^{\prime}} \operatorname{Diag}\left(\lambda_{r_{1}+\theta+1}, \ldots, \lambda_{r}\right) \mathbf{W}^{-}$are positive definite quadratic forms with $\mathbf{W}^{+}=\left(W_{1}+n_{1} / \lambda_{1}, \ldots, W_{r_{1}}+n_{r_{1}} / \lambda_{r_{1}}\right)^{\prime} \sim C_{r_{1}}\left(\boldsymbol{v}_{1}, I ; \xi\right), \boldsymbol{v}_{1}=\left(n_{1} / \lambda_{1}, \ldots, n_{r_{1}} / \lambda_{r_{1}}\right)^{\prime}, \mathbf{W}^{-}=$ $\left(W_{r_{1}+\theta+1}+n_{r_{1}+\theta+1} / \lambda_{r_{1}+\theta+1}, \ldots, W_{r}+n_{r} / \lambda_{r}\right)^{\prime} \sim C_{r-r_{1}-\theta}\left(v_{2}, I ; \xi\right), v_{2}=\left(n_{r_{1}+\theta+1} / \lambda_{r_{1}+\theta+1}, \ldots, n_{r} / \lambda_{r}\right)^{\prime}, \theta$ being number of null eigenvalues of $B^{\prime} A B, n_{j}=\frac{1}{2} m_{j}+b_{j}^{*}, c_{1}=\boldsymbol{\mu}_{1}^{\prime} A \boldsymbol{\mu}_{1}+\mathbf{a}^{\prime} \boldsymbol{\mu}_{1}+d, \kappa_{1}=\left(c_{1}-\sum_{j=1}^{r_{1}} n_{j}^{2} / \lambda_{j}-\sum_{j=r_{1}+\theta+1}^{r} n_{j}^{2} / \lambda_{j}\right)$ and 
$T_{1}=\left(2 \sum_{j=r_{1}+1}^{r_{1}+\theta} n_{j} W_{j}+\kappa_{1}\right) \sim C_{1}\left(\kappa_{1}, 4 \sum_{j=r_{1}+1}^{r_{1}+\theta} n_{j}^{2} ; \xi\right)$. If $\operatorname{rank}(A \Sigma)=\operatorname{rank}(\Sigma)=r, T_{1}=\kappa_{1}$. Note that when $\boldsymbol{\alpha}=\mathbf{0}$ and $\boldsymbol{\mu}=\mathbf{0}$ (the central case), $\boldsymbol{\mu}_{1}=\mathbf{0}$ and $\mathbf{b}^{*}=\mathbf{0}$.

As a particular case, when $\boldsymbol{\alpha}=\mathbf{0}, \mathbf{a}=\mathbf{0}^{\prime}$ and $d=0$, one has the following decomposition for the quadratic form $\mathbf{X}^{\prime} A \mathbf{X}$ in the possibly singular elliptically contoured vector $\mathbf{X} \sim C_{p}(\mu, \Sigma ; \xi), \Sigma$ being of rank $r \leq p$ :

$$
\begin{aligned}
Q(\mathbf{X})= & \mathbf{X}^{\prime} A \mathbf{X}=\sum_{j=1}^{r} \lambda_{j} W_{j}^{2}+2 \sum_{j=1}^{r} b_{j}^{*} W_{j}+c \\
= & \sum_{j=1}^{r_{1}} \lambda_{j} W_{j}^{2}+2 \sum_{j=1}^{r_{1}} b_{j}^{*} W_{j}-\sum_{j=r_{1}+\theta+1}^{r}\left|\lambda_{j}\right| W_{j}^{2}+2 \sum_{j=r_{1}+\theta+1}^{r} b_{j}^{*} W_{j} \\
& +2 \sum_{j=r_{1}+1}^{r_{1}+\theta} b_{j}^{*} W_{j}+c \\
= & \sum_{j=1}^{r_{1}} \lambda_{j}\left(W_{j}+\frac{b_{j}^{*}}{\lambda_{j}}\right)^{2}-\sum_{j=r_{1}+\theta+1}^{r}\left|\lambda_{j}\right|\left(W_{j}+\frac{b_{j}^{*}}{\lambda_{j}}\right)^{2}+2 \sum_{j=r_{1}+1}^{r_{1}+\theta} b_{j}^{*} W_{j} \\
& +\left(c-\sum_{j=1}^{r_{1}} \frac{b_{j}^{* 2}}{\lambda_{j}}-\sum_{j=r_{1}+\theta+1}^{r} \frac{b_{j}^{* 2}}{\lambda_{j}}\right) \\
\equiv & Q_{1}\left(\mathbf{W}_{1}\right)-Q_{2}\left(\mathbf{W}_{2}\right)+2 \sum_{j=r_{1}+1}^{r_{1}+\theta} b_{j}^{*} W_{j}+\kappa \\
\equiv & Q_{1}\left(\mathbf{W}_{1}\right)-Q_{2}\left(\mathbf{W}_{2}\right)+T,
\end{aligned}
$$

where $\mathbf{W}^{\prime}=\left(W_{1}, \ldots, W_{r}\right) \sim \mathcal{S}_{r}(\xi), Q_{1}\left(\mathbf{W}_{1}\right)=\mathbf{W}_{1}^{\prime} \operatorname{Diag}\left(\lambda_{1}, \ldots, \lambda_{r_{1}}\right) \mathbf{W}_{1}$ and $Q_{2}\left(\mathbf{W}_{2}\right)=\mathbf{W}_{2}^{\prime} \operatorname{Diag}\left(\lambda_{r_{1}+\theta+1}, \ldots, \lambda_{r}\right) \mathbf{W}_{2}$ are positive definite quadratic forms with $\mathbf{W}_{1}=\left(W_{1}+b_{1}^{*} / \lambda_{1}, \ldots, W_{r_{1}}+b_{r_{1}}^{*} / \lambda_{r_{1}}\right)^{\prime} \sim C_{r_{1}}\left(v_{1}, I ; \xi\right), v_{1}=\left(b_{1}^{*} / \lambda_{1}, \ldots\right.$, $\left.b_{r_{1}}^{*} / \lambda_{r_{1}}\right)^{\prime}, \mathbf{W}_{2}=\left(W_{r_{1}+\theta+1}+b_{r_{1}+\theta+1}^{*} / \lambda_{r_{1}+\theta+1}, \ldots, W_{r}+b_{r}^{*} / \lambda_{r}\right)^{\prime} \sim C_{r-r_{1}-\theta}\left(\boldsymbol{v}_{2}, I ; \xi\right), v_{2}=\left(b_{r_{1}+\theta+1}^{*} / \lambda_{r_{1}+\theta+1}, \ldots, b_{r}^{*} / \lambda_{r}\right)^{\prime}, \theta$ is the number of null eigenvalues of $A \Sigma$, the $\lambda_{j}$ 's and $b_{j}^{*}$ 's being as previously defined, $c=\mu^{\prime} A \mu, \kappa=(c-$ $\left.\sum_{j=1}^{r_{1}} b_{j}^{* 2} / \lambda_{j}-\sum_{j=r_{1}+\theta+1}^{r} b_{j}^{* 2} / \lambda_{j}\right)$ and $T=2 \sum_{j=r_{1}+1}^{r_{1}+\theta} b_{j}^{*} W_{j}+\kappa \sim C_{1}\left(\kappa, 4 \sum_{j=r_{1}+1}^{r_{1}+\theta} b_{j}^{* 2}\right)$, whenever $\operatorname{rank}(A \Sigma)=r-\theta, \theta=$ $1, \ldots, r-1$. When $\operatorname{rank}(\Sigma)=\operatorname{rank}(A \Sigma)=r, T=\kappa$.

\section{Elliptically Contoured Distributions as Scale Mixtures of Gaussian Vectors}

Normal scale mixtures have the stochastic representation $\mu+\Sigma^{1 / 2} L \mathbf{Z}$, where $\boldsymbol{\mu}$ is the mean of the distribution, $\Sigma^{1 / 2}$ is such that $\Sigma^{1 / 2}\left(\Sigma^{1 / 2}\right)^{\prime}=\Sigma$, the positive semidefinite scale parameter matrix of the distribution, $\mathbf{Z}$ is a standard Gaussian random vector, and $L$ is a positive random variable that is distributed independently of $\mathbf{Z}$. When $\Sigma$ is positive definite, the density function of $\mathbf{Y} \sim \mathcal{C}_{p}(\boldsymbol{\mu}, \Sigma ; \xi)$ can be expressed in terms of a scale mixture of normal densities as follows:

$$
g(\mathbf{y})=\frac{1}{(2 \pi)^{p / 2}|\Sigma|^{1 / 2}} \int_{0}^{\infty} r^{-p / 2} \exp \left\{-\frac{(\mathbf{y}-\boldsymbol{\mu})^{\prime} \Sigma^{-1}(\mathbf{y}-\boldsymbol{\mu})}{2 r}\right\} \mathrm{d} U(r)
$$

where $U(\cdot)$, the distribution function of $L^{2}$, is such that $U(0)=0$. This representation can be found, for example, in Muirhead (1982). A result due to Chu (1973) is extended to non-central elliptically contoured distributions in the next theorem. This theorem enables one to express various distributional results involving elliptically contoured vectors in terms of their Gaussian counterparts.

Theorem 1 Let $\mathbf{Y} \sim \mathcal{C}_{p}(\boldsymbol{\mu}, \Sigma ; \xi)$, with $\Sigma>0, h(\mathbf{y})$ denote the density of $\mathbf{y}$ and $f(s)$ be $h(\mathbf{y})$ wherein $(\mathbf{y}-\boldsymbol{\mu})^{\prime} \Sigma^{-1}(\mathbf{y}-$ $\boldsymbol{\mu}) / 2$ is replaced by $s$. Then, when the inverse Laplace transform of $f(s)$ exists, the density of $\mathbf{Y}$ denoted by h(y) has the following integral representation:

$$
h(\mathbf{y})=\int_{0}^{\infty} w(t) \eta_{\mathbf{Y}}\left(\boldsymbol{\mu}, t^{-1} \Sigma\right) \mathrm{d} t
$$

where $\eta_{\mathbf{Y}}\left(\boldsymbol{\mu}, t^{-1} \Sigma\right)$ denotes the density function of a $p$-dimensional Gaussian random vector with mean $\boldsymbol{\mu}$ and covariance matrix $t^{-1} \Sigma$, and the weighting function $w(t)$ is obtained as

$$
w(t)=(2 \pi)^{p / 2}|\Sigma|^{1 / 2} t^{-p / 2} \mathcal{L}^{-1}(f(s)),
$$


$\mathcal{L}^{-1}(f(s))$ representing the inverse Laplace transform of $f(s)$.

In fact, $\mathcal{L}^{-1}(f(s))$ exists whenever $f(s)$ is an analytic function and $f(s)$ is $O\left(s^{-k}\right)$ as $s \rightarrow \infty$ for $k>1$; for additional properties of the Laplace transform and its inverse, one may refer to Gradshteyn and Ryzhik (1980), Chapter 17. It follows from Theorem 1 that an elliptical distribution is completely specified by its mean $\mu$, scale parameter matrix $\Sigma$ and its weighting function $w(t)$, whenever the latter exists. On letting $t=1 / r$ and defining $w(t)$ to be the density function of $1 / L^{2}$, it is seen that (3) and (4) are equivalent. On integrating $h(\mathbf{y})$ as defined in Theorem 1 over $\mathcal{R}^{p}$ and interchanging the order of integration, one can easily establish that $w(t)$ integrates to 1 . Thus, $w(t)$ can be regarded as a weighting function. Explicit representations of $w(t)$ are given in Table 2 for several $p$-dimensional elliptically contoured distributions.

Theorem 1 enables one to determine the distribution of functions of elliptically contoured vectors in terms of their Gaussian counterparts. For instance, let $\mathbf{Y} \sim \mathcal{C}_{p}(\boldsymbol{\mu}, \Sigma ; \xi)$ and its associated weighting function be $w(t)$. Then, the moment-generating function of the non-central quadratic form $\mathbf{Y}^{\prime} A \mathbf{Y}$ can be obtained as follows:

$$
\begin{aligned}
M_{\mathbf{Y}^{\prime} A \mathbf{Y}}(\theta) & =\int_{-\infty}^{\infty} \cdots \int_{-\infty}^{\infty} \int_{0}^{\infty} e^{\theta \mathbf{y}^{\prime} A \mathbf{y}} w(t) \eta_{\mathbf{Y}}\left(\boldsymbol{\mu}, t^{-1} \Sigma\right) \mathrm{d} t \mathrm{~d} \mathbf{y} \\
& =\int_{0}^{\infty} w(t) M_{Q(\mathbf{W})}^{*}(\theta) \mathrm{d} t
\end{aligned}
$$

where

$$
M_{Q(\mathbf{W})}^{*}(\theta)=\int_{-\infty}^{\infty} \cdots \int_{-\infty}^{\infty} e^{\theta \mathbf{y}^{\prime} A \mathbf{y}} \eta_{\mathbf{Y}}\left(\boldsymbol{\mu}, t^{-1} \Sigma\right) \mathrm{d} \mathbf{y}
$$

is the moment-generating function of the quadratic form $Q(\mathbf{W})=\mathbf{W}^{\prime} A \mathbf{W}$ wherein $\mathbf{W} \sim \mathcal{N}_{p}\left(\boldsymbol{\mu}, t^{-1} \Sigma\right)$.

Similarly, the moments of $\mathbf{Y}^{\prime} A \mathbf{Y}$ can be evaluated as follows:

$$
E\left(\mathbf{Y}^{\prime} A \mathbf{Y}\right)^{h}=\int_{0}^{\infty} w(t) E\left[\left(\mathbf{W}^{\prime} A \mathbf{W}\right)^{h}\right] \mathrm{d} t,
$$

where $\mathbf{W} \sim \mathcal{N}_{p}\left(\boldsymbol{\mu}, t^{-1} \Sigma\right)$ and $E\left[\left(\mathbf{W}^{\prime} A \mathbf{W}\right)^{h}\right]$ can be determined from (7).

In general, the moments of a random variable can be obtained from its cumulants by means of a recursive relationship derived in Smith (1995), which can also be deduced for instance from Theorem 3.2b.2 in Mathai and Provost (1992). For example, the $h^{\text {th }}$ moment of $Q(\mathbf{W})=\mathbf{W}^{\prime} A \mathbf{W}$ is given by

$$
E\left(\mathbf{W}^{\prime} A \mathbf{W}\right)^{h}=\mu_{h}=\sum_{i=0}^{h-1} \frac{(h-1) !}{(h-1-i) ! i !} k(h-i) \mu_{i}
$$

where $k(h)$, the $h^{\text {th }}$ cumulant of $Q(\mathbf{W})$, is

$$
k(h)=\left\{\begin{array}{l}
2^{h-1} h !\left(\frac{\operatorname{tr}\left(t^{-1} A \Sigma\right)^{h}}{h}+\mu^{\prime}\left(t^{-1} A \Sigma\right)^{h-1} A \mu\right), \quad h \geq 2, \\
\operatorname{tr}\left(t^{-1} A \Sigma\right)+\mu^{\prime} A \mu, \quad h=1 .
\end{array}\right.
$$

\section{Illustrative Examples}

Four numerical examples involving quadratic forms and quadratic expressions in various types of elliptically contoured vectors are presented in this section. The steps of the proposed methodology for determining their distributions are described in the first example.

Example 1 Consider the quadratic form $Q^{\mathrm{I}}(\mathbf{X})=\mathbf{X}^{\prime} A \mathbf{X}$ where $\mathbf{X}$ has a noncentral $t$-distribution with 10 degrees of freedom whose density function is as given in Table 1 with $s=(\mathbf{x}-\boldsymbol{\mu})^{\prime} \Sigma^{-1}(\mathbf{x}-\boldsymbol{\mu}) / 2, \boldsymbol{\mu}=(0,1,3,2)^{\prime}$,

$$
\Sigma=\left(\begin{array}{cccc}
1 & 1 / 2 & 2 / 5 & 1 / 2 \\
1 / 2 & 1 & 1 / 4 & 3 / 8 \\
2 / 5 & 1 / 4 & 1 & 1 / 3 \\
1 / 2 & 3 / 8 & 1 / 3 & 1
\end{array}\right) \quad \text { and } \quad A=\left(\begin{array}{rrrr}
1 & -6 & 2 & 1 \\
-6 & 7 & 0 & 4 \\
2 & 0 & -4 & 1 \\
1 & 4 & 1 & 2
\end{array}\right)
$$


The proposed methodology comprises the following steps:

1) $Q^{\mathrm{I}}(\mathbf{X})$ is expressed as $Q_{1}^{\mathrm{I}}\left(\mathbf{W}_{1}\right)-Q_{2}^{\mathrm{I}}\left(\mathbf{W}_{2}\right)+\kappa$ in accordance with Equation (2).

2) The moments of $Q_{i}^{\mathrm{I}}\left(\mathbf{W}_{i}\right), i=1,2$ are determined from Equations (6) and (7).

3) A generalized gamma density function,

$$
\psi(z)=\frac{\gamma}{\beta^{\alpha \gamma} \Gamma(\alpha)} z^{\alpha \gamma-1} e^{-(z / \beta)^{\gamma}} \mathcal{I}_{(0, \infty)}(z), \quad \alpha>0, \beta>0, \gamma>0
$$

is taken as base density for $Q_{i}^{\mathrm{I}}\left(\mathbf{W}_{i}\right), i=1,2$.

4) The parameters $\alpha, \beta$ and $\gamma$ are determined by simultaneously solving the following nonlinear equations

$$
\mu_{j}=m_{j} \quad \text { for } j=1,2,3,
$$

where

$$
m_{j}=\frac{\beta^{j} \Gamma(\alpha+j / \gamma)}{\Gamma(\alpha)}, j=0,1, \ldots
$$

are the moments associated with a generalized gamma density function and $\mu_{j}$ can be determined from the recursive formula (7).

5) A polynomial adjustment of degree $d$ can be made as explained in the Appendix, the resulting density approximation being

$$
f_{d}(z)=\psi(z) \sum_{j=0}^{d} \xi_{j} z^{j}
$$

in this case, we set $d=7$.

6) Given the density approximations determined for $Q_{1}^{\mathrm{I}}\left(\mathbf{W}_{1}\right)$ and $Q_{2}^{\mathrm{I}}\left(\mathbf{W}_{2}\right)$, the approximate density of the difference is obtained by applying the transformation of variables technique. Shifting this density by $\kappa$ then yields the desired approximation.

Certain values of the resulting approximate distribution function of $Q^{\mathrm{I}}(\mathbf{X})$ are included in Table 1 . The percentiles were obtained by simulation on the basis of 1,000,000 replications. The plot shown in Figure 1 confirms that the proposed approach yields a very accurate approximation to the distribution of $Q^{\mathrm{I}}(\mathbf{X})$.

Table 1. Approximate cdf of $Q^{\mathrm{I}}(\mathbf{X})$ evaluated at certain percentiles obtained by simulation (Simul\%)

\begin{tabular}{rrc}
\hline$C D F$ & Simul\% & Approx. \\
\hline 0.01 & -93.013 & 0.009429 \\
0.05 & -56.312 & 0.052101 \\
0.10 & -40.539 & 0.101342 \\
0.50 & 7.9373 & 0.509682 \\
0.90 & 77.615 & 0.893073 \\
0.95 & 106.51 & 0.942475 \\
0.99 & 179.76 & 0.988139 \\
\hline
\end{tabular}

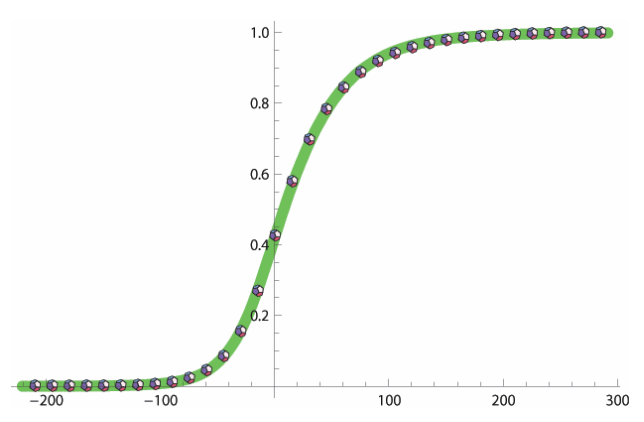

Figure 1. Simulated cdf of $Q^{I}(\mathbf{X})$ and cdf approximation (dots) 
Example 2 Consider the quadratic form $Q^{\mathrm{II}}(\mathbf{X})=\mathbf{X}^{\prime} A \mathbf{X}$ where $\mathbf{X}$ is a contaminated normal random vector as specified in Table 2, for which $\phi=0.4, \boldsymbol{\mu}=(1,2,3)^{\prime}$,

$$
\Sigma=\left(\begin{array}{ccc}
1 & 0.2 & 0.7 \\
0.2 & 1 & 0.2 \\
0.7 & 0.2 & 1
\end{array}\right) \quad \text { and } \quad A=\left(\begin{array}{rrr}
5 & 3 & 2 \\
3 & -5 & 5 \\
2 & 5 & -2
\end{array}\right) \text {. }
$$

It this case, a gamma distribution (as defined by (8) with $\gamma=1$ ) was utilized as base density to obtain an approximate distribution for each quadratic form in decomposition of $Q^{\mathrm{II}}(\mathbf{X})$. Letting the integer moments of a non-negative definite quadratic form be denoted by $\mu_{j}, j=1,2, \ldots$, a gamma approximation can be specified by equating its first two moments to $\mu_{1}$ and $\mu_{2}$, respectively, and solving for $\alpha$ and $\beta$, that is, $\alpha \beta=\mu_{1}$ and $\alpha(\alpha+1) \beta^{2}=\mu_{2}$, which yields

$$
\alpha=\frac{\mu_{1}^{2}}{\mu_{2}-\mu_{1}^{2}} \text { and } \beta=\frac{\mu_{2}}{\mu_{1}}-\mu_{1} .
$$

The methodology described in Example 1 was applied in conjunction with polynomial adjustments of degree six to determine the approximate distribution of $Q^{\mathrm{II}}(\mathbf{X})$. The plot shown in Figure 2 indicates that the resulting approximation is very accurate.

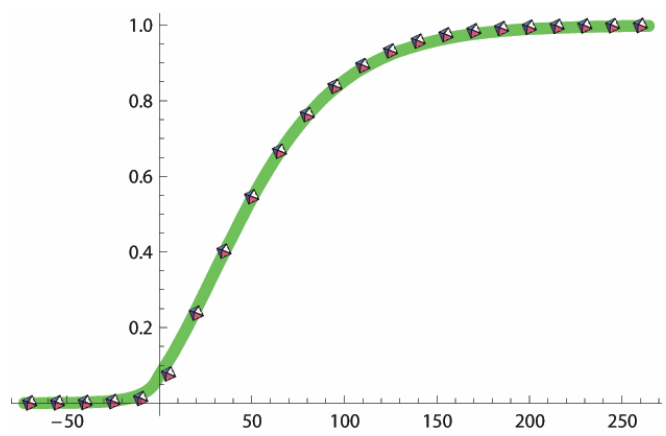

Figure 2. Simulated cdf of $Q^{\mathrm{II}}(\mathbf{X})$ and cdf approximation (dots)

Table 2. Some elliptically contoured distributions and their weighting functions

\begin{tabular}{lll}
\hline Distribution & \multicolumn{1}{c}{ Density function } & Weighting function \\
\hline Gaussian & $e^{-s} /\left((2 \pi)^{p / 2}|\Sigma|^{1 / 2}\right)$ with & $\delta(t-1)$ \\
& $s=\mathbf{x}^{\prime} \Sigma^{-1} \mathbf{x} / 2$ throughout & The Dirac delta function \\
Contaminated Normal & $\left\{\phi \lambda^{p / 2} e^{-\lambda s}+(1-\phi) e^{-s}\right\} /$ & $\phi \delta(t-\lambda)+(1-\phi) \delta(t-1)$ \\
& $\left\{(2 \pi)^{p / 2}|\Sigma|^{1 / 2}\right\}$ & \\
$t$-distribution with $v$ d.f. & $\left\{v^{v / 2} \Gamma((v+p) / 2)|\Sigma|^{-1 / 2}\right.$ & $\left\{v(v t / 2)^{(v / 2)-1} e^{-v t / 2}\right\} /\{2 \Gamma(v / 2)\}$ \\
& $\left.\times(v+2 s)^{-(v+p) / 2}\right\} /\left\{\pi^{p / 2} \Gamma(v / 2)\right\}$ & \\
Multivariate Analog of the & $\left\{\Gamma(p / 2) e^{-\sqrt{2 s}\} /}\right.$ & $\left\{\Gamma(p / 2) e^{-1 / 2 t}\right\} /\{\Gamma(p)$ \\
Bilateral Exponential Density & $\left\{2 \pi^{p / 2} \Gamma(p)|\Sigma|^{1 / 2}\right\}$ & $\left.\times 2^{(3-p) / 2} \sqrt{\pi} t^{(p+3) / 2}\right\}$ \\
The Generalized Slash Distribution & $v s^{-p / 2-v}|\Sigma|^{-1 / 2} \times\{\Gamma(p / 2+v)$ & $\left\{v t^{v-1}, 0<v<1\right.$ \\
& $-\Gamma(p / 2+v, s)\} /(2 \pi)^{p / 2}$ & $v$, \\
& &
\end{tabular}

Example 3 Consider the quadratic form $Q^{\mathrm{II}}(\mathbf{X})=\mathbf{X}^{\prime} A \mathbf{X}$ where $\mathbf{X}$ follows a generalized slash distribution whose density function is as defined in Table 2 with $\boldsymbol{\mu}=(0,1,2)^{\prime}$,

$$
\Sigma=\left(\begin{array}{ccc}
1 & 1 / 2 & 2 / 5 \\
1 / 2 & 1 & 1 / 4 \\
2 / 5 & 1 / 4 & 1
\end{array}\right) \quad \text { and } \quad A=\left(\begin{array}{rrr}
1 & -6 & 2 \\
-6 & 7 & 0 \\
2 & 0 & -4
\end{array}\right)
$$


By making use of the weighting function associated with the generalized slash distribution in order to determine the moments (Equation (7)) of the quadratic forms occurring in its decomposition and implementing the steps described in Example 1 in conjunction with a gamma distribution or a generalized gamma distribution whose associated densities are taken as base densities, one can determine an approximate distribution for $Q^{\mathrm{III}}(\mathbf{X})$.

The left and right panels of Figure 3 respectively show the distribution functions resulting from gamma and generalized gamma approximations, which are superimposed on the simulated distribution function determined on the basis of 1,000,000 replications.
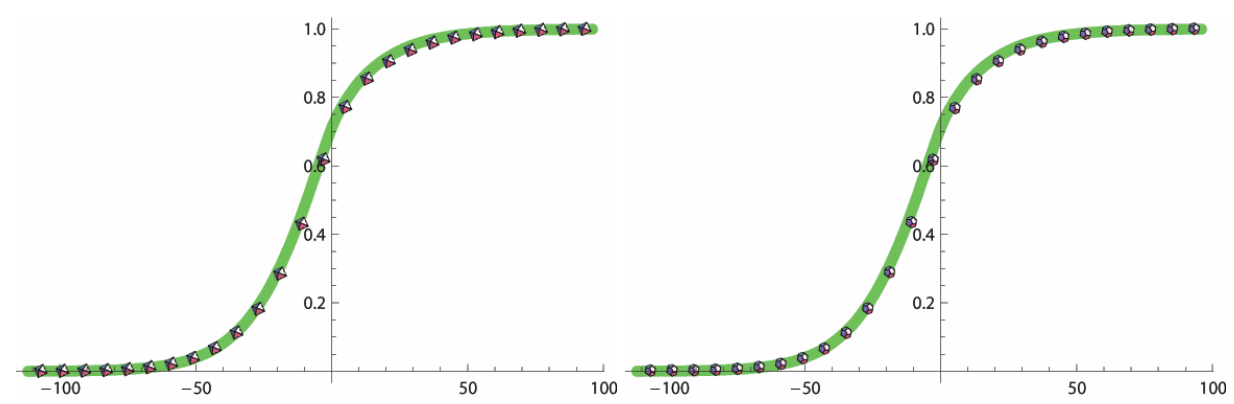

Figure 3. Simulated cdf of $Q^{\mathrm{III}}(\mathbf{X})$ and approximations based on polynomially adjusted gamma (left panel) and generalized gamma (right panel) distributions (dots)

Example 4 Let $Q_{1}^{*}(\mathbf{X})=(\mathbf{X}-\boldsymbol{\alpha})^{\prime} A(\mathbf{X}-\boldsymbol{\alpha})+\mathbf{a}^{\prime}(\mathbf{X}-\boldsymbol{\alpha})+d$ be a quadratic expression in a singular $t-$ vector with 10 degrees of freedom where $\mathbf{X} \sim C_{5}(\boldsymbol{\mu}, \Sigma ; \xi), \boldsymbol{\mu}=(4,1,-1,3,2)^{\prime}$,

$$
\Sigma=\left(\begin{array}{lllll}
3 & 3 & 3 & 2 & 0 \\
3 & 3 & 3 & 2 & 0 \\
3 & 3 & 5 & 2 & 0 \\
2 & 2 & 2 & 2 & 0 \\
0 & 0 & 0 & 0 & 1
\end{array}\right)
$$

which is singular, $\alpha=(1,1,0,1,1)^{\prime}, A$ is the following indefinite matrix

$$
A=\left(\begin{array}{rrrrr}
1 & 1 & 2 & 3 & -5 \\
1 & 1 & 2 & 3 & -5 \\
2 & 2 & 0 & 0 & 0 \\
3 & 3 & 0 & 0 & 0 \\
-5 & -5 & 0 & 0 & -26
\end{array}\right)
$$

$\mathbf{a}=(1,2,3,4,5)^{\prime}$ and $d=6$.

When $\Sigma_{p \times p}$ is a singular matrix of rank $r<p$, we make use of the spectral decomposition theorem to express $\Sigma$ as $U W U^{\prime}$ where $W$ is a diagonal matrix whose first $r$ diagonal elements are positive, the remaining diagonal elements being equal to zero. Next, we let $B_{p \times p}^{*}=U W^{1 / 2}$ and remove the last $p-r$ columns of $B^{*}$, which are null vectors, to obtain the matrix $B_{p \times r}$. Then, it can be verified that $\Sigma=B B^{\prime}$. In this case, the matrices $B$ and $P$ were found to be

$$
B=\left(\begin{array}{cccc}
1.66591 & 0.39015 & 0 & -0.26930 \\
1.66591 & 0.39015 & 0 & -0.26930 \\
2.03287 & -0.92672 & 0 & 0.09291 \\
1.18171 & 0.49418 & 0 & 0.59945 \\
0 & 0 & 1 & 0
\end{array}\right)
$$

and

$$
P=\left(\begin{array}{rrrr}
-0.97731 & 0.00042 & -0.14936 & -0.15022 \\
0.05695 & -0.58347 & -0.72923 & 0.35290 \\
0.13922 & 0.69384 & -0.66277 & -0.24484 \\
-0.14916 & 0.42208 & 0.08157 & 0.89048
\end{array}\right)
$$


respectively. One can utilize the decomposition of $Q^{*}(\mathbf{X})$, which is provided in Equation (1), to determine an approximation to the distribution function of $Q_{1}^{*}(\mathbf{X})$. The approximate density functions of $Q_{1}\left(\mathbf{W}^{+}\right)$and $Q_{2}\left(\mathbf{W}^{-}\right)$ are obtained by making use of a gamma approximation, as explained in Example 2. We first apprximated density of $Q_{1}\left(\mathbf{W}^{+}\right)-Q_{2}\left(\mathbf{W}^{-}\right)$and then, determined the density function of $Q_{1}\left(\mathbf{W}^{+}\right)-Q_{2}\left(\mathbf{W}^{-}\right)+T_{1}$ by applying the transformation of variables technique.

Referring again to the decomposition (1), the eigenvalues of $B^{\prime} A B$ were found to be $\lambda_{1}=65.8197, \lambda_{2}=-29.5759$, $\lambda_{3}=-2.24383, \lambda_{4}=0$, and it was determined that $n_{1}=-43.6247, n_{2}=31.6913, n_{3}=2.87613$, and $n_{4}=-0.154303$, and that $\mu_{1}=-0.662791, \mu_{2}=(-1.07153,-1.2818)^{\prime}$ and $c_{1}=-4$. The resulting distribution function was evaluated at certain simulated percentiles obtained on the basis of 500,000 replications. The results are presented in Table 3 and the approximate cdf is plotted in Figure 4.

Table 3. Approximate cdf of $Q_{1}^{*}(\mathbf{X})$ evaluated at certain percentiles obtained by simulation (Simul\%)

\begin{tabular}{rrc}
\hline$C D F$ & Simul\% & Approx. \\
\hline 0.01 & -364.29 & 0.011478 \\
0.05 & -188.33 & 0.058634 \\
0.10 & -123.21 & 0.110176 \\
0.50 & 6.1069 & 0.506286 \\
0.90 & 233.11 & 0.893207 \\
0.95 & 360.83 & 0.943116 \\
0.99 & 722.56 & 0.988926 \\
\hline
\end{tabular}

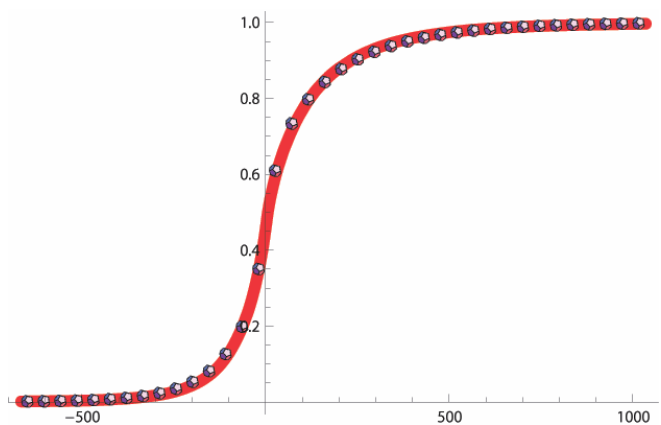

Figure 4. Simulated cdf of $Q_{1}^{*}(\mathbf{X})$ and approximation (dots)

\section{Appendix}

\section{A.1 Polynomially Adjusted Gamma Density Approximations}

As explained in Provost (2005), the density functions of numerous statistics distributed on the positive half-line can be approximated from their exact moments by means of gamma-type density functions that are adjusted with linear combinations of Laguerre polynomials. For conditions ensuring that a distribution be uniquely defined by its moments, the reader is referred to Rao (1965).

Consider a random variable $Y$ defined on the interval $[0, \infty)$, whose $j^{\text {th }}$ moment is denoted by $\mu_{j}, j=0,1,2, \ldots$, and let $c=\left(\mu_{2}-\mu_{1}^{2}\right) / \mu_{1}, v=\left(\mu_{1} / c\right)-1$ and $X=Y / c$. Denoting the $j^{\text {th }}$ moment of $X$ by $\mu_{j}^{*}=E\left[(Y / c)^{j}\right]$, the density function of the random variable $X$, also defined on the interval $[0, \infty)$, can be expressed as

$$
f(x)=x^{v} e^{-x} \sum_{j=0}^{\infty} \delta_{j} L_{j}(v, x)
$$

where

$$
L_{j}(v, x)=\sum_{k=0}^{j}(-1)^{k} \frac{\Gamma(v+j+1) x^{j-k}}{k !(j-k) ! \Gamma(v+j-k+1)}
$$


is a Laguerre polynomial of order $j$ in $x$ with parameter $v$ and

$$
\delta_{j}=\sum_{k=0}^{j}(-1)^{k} \frac{j !}{k !(j-k) ! \Gamma(v+j-k+1)} \mu_{j-k}^{*},
$$

see for instance Szegö (1959) or Devroye (1989). Then, on truncating the series appearing in Equation (10) and making the change of variable $Y=c X$, one obtains the following density approximant for $Y$ :

$$
f_{d}(y)=\frac{y^{v} e^{-y / c}}{c^{v+1}} \sum_{j=0}^{d} \delta_{j} L_{j}(v, y / c) .
$$

Remark A.1 Note that $f_{0}(y)$ is a gamma density function with parameters $\alpha \equiv v+1=\mu_{1}^{2} /\left(\mu_{2}-\mu_{1}^{2}\right)$ and $\beta \equiv c=$ $\left(\mu_{2}-\mu_{1}^{2}\right) / \mu_{1}$ whose mean, $\alpha \beta=\mu_{1}$, and variance, $\alpha \beta^{2}=\mu_{2}-\mu_{1}^{2}$, match the mean and variance of $Y$ and that, in light of Equation (13), one can express $f_{d}(y)$ as the product of an initial gamma density approximation specified by $f_{0}(y)$ times a polynomial adjustment:

$$
f_{d}(y)=\frac{y^{\alpha-1} e^{-y / \beta}}{\beta^{\alpha} \Gamma(\alpha)} \sum_{j=0}^{d} \omega_{j} L_{j}\left(\alpha-1, \frac{y}{\beta}\right),
$$

where $\omega_{j}=\Gamma(\alpha) \delta_{j}$.

\section{References}

Cambanis, S., Huang, S., \& Simmons, G. (1981). On the theory of elliptically contoured distributions. Journal of Multivariate Analysis, 11, 368-385. http://dx.doi.org/10.1016/0047-259X(81)90082-8

Chmielewski, M. A. (1981). Elliptically symmetric distributions: A review and bibliography. International Statistical Review, 49, 67-74. http://dx.doi.org/10.2307/1403038

Chu, K.-U. (1973). Estimation and decision for linear systems with elliptically random process. IEEE Transaction on Automatic Control, 18, 499-505. http://dx.doi.org/10.1109/TAC.1973.1100374

Devlin, S. J., Gnanadesikan, R., \& Kettenring, J. R. (1976). Some Multivariate Applications of Elliptical Distributions. Essays in Probability and Statistics (pp. 365-393), Ed. S. Ideka, . Sinko Tsusho, Tokyo.

Devroye, L. (1989). On random variate generation when only moments or Fourier coefficients are known. Mathematics and Computers in Simulation, 31, 71-89. http://dx.doi.org/10.1016/0378-4754(89)90054-2

Díaz-García, J. A., \& Leiva-Sánchez, V. (2005). A new family of life distributions based on the elliptically contoured distributions. Journal of Statistical Planning and Inference, 128, 445-457. http://dx.doi.org/10.1016/j.jspi.2003.11.007

Fama, E. F. (1965). The behavior of stock-market prices. Journal of Business, XXXVIII, 34-105. http://dx.doi.org/10.1086/294743

Fang, K.-T., Kotz, S., \& Ng, K.-W. (1990). Symmetric Multivariate and Related Distributions. London: Chapman and Hall.

Fraser, D. A. S., \& Ng, K.-W. (1980). Multivariate regression analysis with spherical error. In Multivariate Analysis 5 (pp. 369-386), Ed. P. R. Krishnaiah. New York: North Holland.

Girón, F. J., \& Rojano, J. C. (1994). Bayesian Kalman filtering with elliptically contoured errors. Biometrika, 80, 390-395. http://dx.doi.org/10.1093/biomet/81.2.390

Gradshteyn, I. S., \& Ryzhik, I. M. (1980). Table of Integrals, Series, and Products, Corrected and Enlarged Edition. New York: Academic Press Inc.

Hamada, M., \& Valdez, E. A. (2008). CAPM and option pricing with elliptically contoured distributions. Journal of Risk \& Insurance, 75(2), 387-409. http://dx.doi.org/10.1111/j.1539-6975.2008.00265.x

Ip, W. C., Wonga, H., \& Liu, J. S. (2007). Inverse Wishart distributions based on singular elliptically contoured distribution. Linear Algebra and its Applications, 420, 424-432. http://dx.doi.org/10.1016/j.laa.2006.07.027 
Kelker, D. (1970). Distribution theory of spherical distributions and a location-scale parameter generalization. Sankhyā Series A, 32, 419-430.

King, M. L. (1980). Robust tests for spherical symmetry and their application to least squares regression. Annals of Statistics, 8, 1265-1271. http://dx.doi.org/10.1214/aos/1176345199

Mandelbrot, B. (1963). The variation of certain speculative prices. Journal of Business, XXXVI, 394-419. http://dx.doi.org/10.1086/294632

Mathai, A. M., \& Provost, S. B. (1992). Quadratic Forms in Random Variables, Theory and Applications. New York: Marcel Dekker Inc.

Mathai, A. M., Provost, S. B., \& Hayakawa, T. (1995). Bilinear Forms and Zonal Polynomials. New York: Springer-Verlag. http://dx.doi.org/10.1007/978-1-4612-4242-0

McGraw, D. K., \& Wagner, J. F. (1968). Elliptically symmetric distributions. IEEE Trans. on Information Theory, 14, 110-120. http://dx.doi.org/10.1109/TIT.1968.1054081

Muirhead, R. J. (1982). Aspects of Multivariate Statistical Theory. New York: Wiley. http://dx.doi.org/10.1002/9780470316559

Osiewalski, J., \& Steel, M. F. J. (1993). Robust Bayesian inference in elliptical regression models. Journal of Econometrics, 57, 345-363. http://dx.doi.org/10.1016/0304-4076(93)90070-L

Resnick, S. I. (1997). Heavy tail modeling and teletraffic data. The Annals of Statistics, 25, 1805-1869. http://dx.doi.org/10.1214/aos/1069362376

Smith, P. J. (1995). A recursive formulation of the old problem of obtaining moments from cumulants and vice versa. The American Statistician, 49, 217-219. http://dx.doi.org/10.1080/00031305.1995.10476146

Szegö, G. (1959). Orthogonal Polynomials. RI: American Mathematical Society.

Theiler, J., Scovel, C., Wohlberg, B., \& Foy, B. (2010). Elliptically contoured distributions for anomalous change detection in hyperspectral imagery. IEEE Geoscience and Remote Sensing Letters, 7, 271-275. http://dx.doi.org/10.1109/LGRS.2009.2032565

Zellner, A. (1976). Bayesian and non-Bayesian analysis of the regression model with multivariate Student- $t$ error terms. Journal of American Statistical Association, 71, 400-405. http://dx.doi.org/10.1080/01621459.1976.10480357 